\title{
Specific Cleavage of 28S Ribosomal RNA in Murine Coronavirus-Infected Cells
}

\author{
${ }^{1,2}$ SANGEETA BANERJEE, ${ }^{1}$ SUNGWHAN AN, AND ${ }^{1,2}$ SHINJI MAKINO \\ 'Department of Microbiology and Institute of Cellular and Molecular Biology, The University of \\ Texas at Austin, Austin, Texas 78712: ${ }^{2}$ Department of Microbiology and Immunology, The \\ University of Texas Medical Branch, Galveston, Texas 77555
}

\section{INTRODUCTION}

Mouse hepatitis virus (MHV), a prototypic coronavirus, causes gastrointestinal and upper respiratory tract illnesses in animals and man. The $32 \mathrm{~kb}$-long MHV genome is a positive-sense, single-stranded RNA (Lai et al 1981, Lee et al 1991) that encodes 11 open reading frames, expressed through the production of a genomic-size and six to eight species of subgenomic mRNAs (Lai et al 1981). Genomic-size mRNA encodes the 5'most $22 \mathrm{~kb}$-long gene 1, which encodes the RNA polymerase function (Lee et al 1991). MHV contains three envelope proteins, S, M and E proteins. S protein binds to the coronavirus receptor (Dveskler et al 1991). $\mathrm{M}$ and $\mathrm{E}$ proteins are important for the formation of the MHV envelope (Kim et al 1997).

Extensive morphological and physiological changes occur in coronavirus-infected cells. Some of these changes contribute to damage of cells and tissues. The specific basis, for these deleterious effects on host cells, is not well understood. MHV-infection causes host protein translation inhibition (Hilton et al 1986, Tahara et al 1994). Inhibition of host protein synthesis accompanies an increase in MHV protein synthesis (Siddell et al 1981). Some host mRNAs are degraded in MHV-infected cells, while others are transcriptionally upregulated (Kyuwa et al 1994). The mechanism of selective MHV-specific protein synthesis in infected cells, concomitant with

The Nidoviruses (Coronaviruses and Arteriviruses).

Edited by Ehud Lavi et al., Kluwer Academic/Plenum Publishers, 2001 
host protein inhibition, is poorly characterised, although it has been suggested that binding of $\mathrm{N}$ protein to MHV leader sequences may act as a strong translation initiation signal (Tahara et al 1994, Tahara et al 1998).

In the present study, we described a hitherto unknown phenomenon in MHV-infected cells: the specific cleavage of 28S ribosomal RNA (rRNA). This phenomenon was not restricted to any particular MHV strain or host cell type. Our data also suggested that the observed cleavage was probably not regulated by the apoptotic pathway. Hence we demonstrated that MHVinduced 28 rRNA cleavage was different from other known ribosomal RNA cleavage events.

\section{MATERIALS AND METHODS}

\subsection{Viruses and cells}

The plaque-cloned A59 strain of MHV (Lai et al 1981), MHV-JHM and MHV-2 were used. Mouse DBT cells were used for MHV growth.

\section{$2.2 \quad$ Northern (RNA) blotting}

Northern blot analysis was performed using $\left[{ }^{32} \mathrm{P}\right]-\gamma$ ATP-labeled oligonucleotide probes as previously described (Makino et al 1991). Probe 1 binding to nucleotides 1532-1551 from the 5'-end of mouse 28S rRNA, was used to detect $28 \mathrm{~S}$ rRNA and its cleavage products. Probe 2 and probe 3 , binding to nucleotides 921-940 and nucleotides 1846-1870 from the 5'-end of mouse 18S rRNA, respectively, were used to detect $18 \mathrm{~S}$ rRNA. All hybridisation were performed at $60^{\circ} \mathrm{C}$.

\section{RESULTS}

Agarose gel electrophoresis of intracellular RNA from MHV-A59infected DBT cells showed a major band that migrated between the $28 \mathrm{~S}$ and 18S rRNAs. No MHV-specific probe hybridised with this RNA band and its size differed from all MHV subgenomic mRNAs (data not shown). Northern blot analysis of intracellular RNA from MHV-infected cells, using a 28S rRNA-specific probe 1 (Fig. 1A) revealed a major 28S rRNA cleavage product, 28S-CL1 and four minor 28S rRNA bands, CL2-CL5, in addition to the intact $28 \mathrm{~S}$ rRNA. $28 \mathrm{~S}-\mathrm{CL} 1$ appeared as early as $4 \mathrm{~h}$ p.i. (data not shown) 
and it remained a major cleavage product until $12 \mathrm{~h}$ p.i. $28 \mathrm{~S}-\mathrm{CL}-3$ and $28 \mathrm{~S}-$ CL4 accumulated late in infection. The amount of intact 28S rRNA decreased significantly with increasing time of infection. In contrast, $18 \mathrm{~S}$ rRNA did not undergo cleavage in MHV-infected DBT cells (Fig. 1B). The amount of $18 \mathrm{~S}$ rRNA in MHV-A59-infected cells remained similar to that in uninfected cells, until $16 \mathrm{~h}$ p.i., when it decreased slightly.

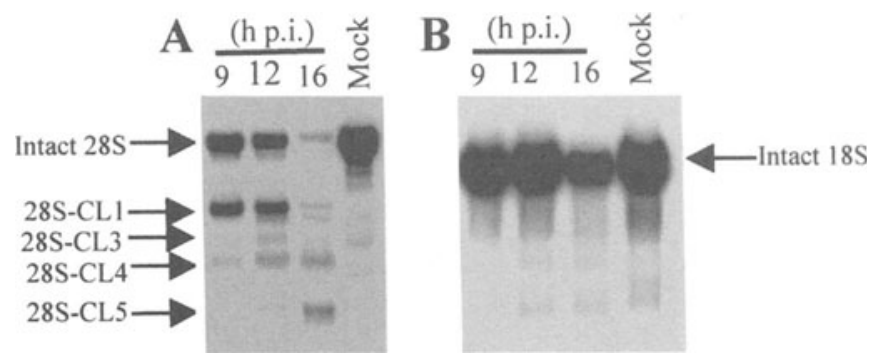

Figure 1. Specific degradation of 28S rRNA in MHV-infected DBT cells. Cytoplasmic RNA was extracted from MHV-infected cells at indicated times and Northern blot analysis was performed using oligonucleotide probes against mouse 28S rRNA (A) and 18S rRNA (B).

Infecting DBT cells with UV-inactivated MHV inoculum did not cause any $28 \mathrm{~S}$ rRNA cleavage (Fig. 2A), demonstrating that binding of MHV to MHV receptors alone or other unidentified substances, did not induce $28 \mathrm{~S}$ rRNA cleavage. Induction of $28 \mathrm{~S}$ rRNA cleavage required MHV replication. Comparing the kinetics of $28 \mathrm{~S}$ rRNA cleavage with other cytopathic effects in MHV-infected cells, we found 28S rRNA cleavage started earlier than the onset of MHV-A59-induced cell fusion. Infection with a nonfusogenic MHV strain, MHV-2, also caused 28S rRNA cleavage, in DBT cells (data not shown). These data demonstrated that MHV-induced 28S rRNA cleavage was not directly related to cell fusion. A substantial increase in the amount of 28S-CL1, in MHV-infected cells, preceded the peak of MHV RNA synthesis and the cleavage continued at a time when MHV RNA synthesis declined (Sawicki and Sawicki 1986).

We examined the relationship between 28S rRNA cleavage and host protein synthesis in MHV-infected cells. Consistent with previous studies (Siddell et al 1981, Tahara et al 1994), host protein synthesis inhibition in MHV-infected cells was evident by $6 \mathrm{~h}$ p.i. (data not shown); 28S-CL1 appeared as a major cleavage product starting $5 \mathrm{~h}$ p.i. Hence, the $28 \mathrm{~S}-\mathrm{CL} 1$ cleavage product needed to accumulate before host protein synthesis inhibition could occur. Thereafter, both $28 \mathrm{~S}$ rRNA cleavage and host protein synthesis inhibition continued as infection proceeded. 

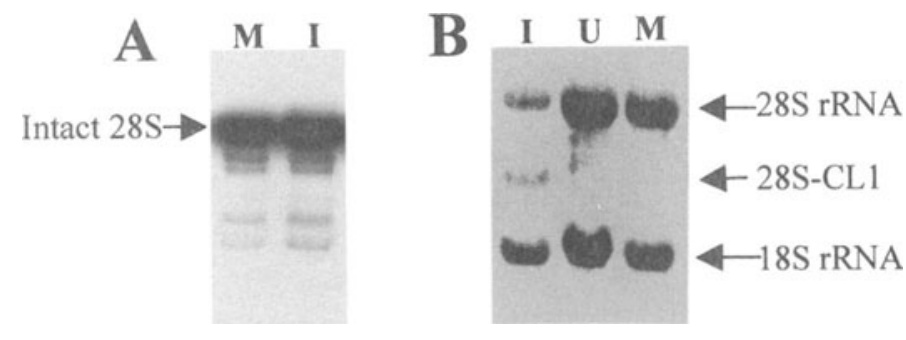

Figure 2. MHV replication is required for mature cytoplasmic 28S rRNA cleavage. (A) Cytoplasmic RNA from mock-infected (M) or UV-irradiated MHV-A59-infected (I) DBT cells were extracted at $8 \mathrm{~h}$ p.i. and analysed by Northern blot. (B) Ribosomal precursors were labelled with ${ }^{3} \mathrm{H}$-uridine and chased for $13 \mathrm{hr}$. Then, cells were mock-infected $(\mathrm{M})$ or infected with MHV-A59 (I). $8 \mathrm{~h}$ p.i. cytoplasmic RNA was extracted. $\mathrm{U}=$ Uninfected.

To determine whether mature cytoplasmic rRNA is cleaved in MHVinfected cells, precursor rRNA molecules, in DBT cells, were labelled with ${ }^{3} \mathrm{H}$-uridine and allowed to be processed and transported to the cytoplasm. Cells were then infected with MHV-A59 and labelled, mature cytoplasmic RNA were analysed by electrophoresis (Fig. 2B). Mock-infected cells showed intact $28 \mathrm{~S}$ rRNAs while MHV-infected cells showed a reduced amount of intact $28 \mathrm{~S}$ rRNA and the presence of 28S-CL1. We concluded that MHV infection induced cleavage of mature $28 \mathrm{~S}$ rRNA, that was processed and transported to the cytoplasm, prior to MHV infection.

28S rRNA cleavage was not confined to any particular cell type or MHV strain. MHV-A59-infection to DBT and 17Cl-1 cells produced all 28S rRNA cleavage products; the kinetics of appearance of the cleavage products and reduction of intact 28S rRNA in MHV-A59-infected DBT and 17CL-1 cells were similar (data not shown). MHV-JHM-infection to DBT and $17 \mathrm{Cl}-1$ cells also induced similar 28S rRNA cleavage products but progress of MHV-JHM-induced 28S rRNA cleavage was slow (data not shown); A lower level of MHV-JHM replication efficiency in infected cells may be related to less efficient $28 \mathrm{~S}$ rRNA cleavage.

There have been some reports of rRNA degradation occurring in apoptotic cells. MHV-infected $17 \mathrm{Cl}-1$ cells undergo apoptosis, but not MHV-infected DBT cells (An et al 1999). Nevertheless, 28S rRNA cleavage occurred in both DBT and 17Cl-1 cells (Fig. 1, and data not shown). If apoptosis regulates MHV-induced 28S rRNA cleavage then blocking apoptosis should block 28S rRNA cleavage. Accordingly we treated MHVinfected 17Cl-1 cells with an irreversible caspase-3 inhibitor, Z-DEVD-fmk, that effectively blocks apoptosis. We found that blocking apoptosis in MHV-infected $17 \mathrm{Cl}-1$ cells did not block or delay $28 \mathrm{~S}$ rRNA cleavage (Fig. 3). Thus, MHV-induced $28 \mathrm{~S}$ rRNA cleavage was upstream of caspase- 
activation, or apoptosis induction and 28S rRNA cleavage occurred via two independent pathways.

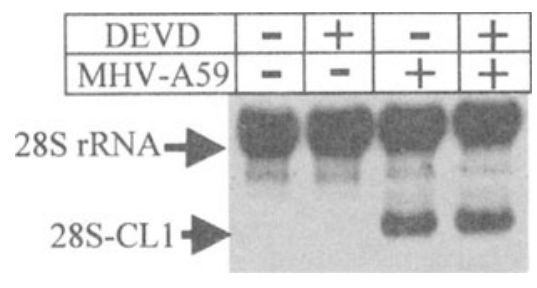

Figure 3. Blocking apoptosis does not block MHV-induced 28S rRNA cleavage. $17 \mathrm{Cl}-1$ cells were mock-treated or treated with $80 \mu \mathrm{M}$ Z-DEVD-fmk and mock-infected or infected with MHV-A59. $8 \mathrm{~h}$ p.i. cytoplasmic RNA was extracted and analyzed by Northern blot.

\section{DISCUSSION}

We report a novel MHV-induced 28S rRNA cleavage in the present study. The MHV-induced rRNA cleavage occurred only in 28S rRNA, and not in 18S rRNA. Specific cleavage of $28 \mathrm{~S}$ rRNA required ongoing MHV replication since UV-inactivated MHV failed to induce $28 \mathrm{~S}$ rRNA cleavage. Fully processed, mature cytoplasmic $28 \mathrm{~S}$ rRNA underwent cleavage. Currently, we do not know which step of the viral life cycle or specific viral factor(s) causes this $28 \mathrm{~S}$ rRNA cleavage. MHV infection to all susceptible cell lines, using several different MHV strains, induced 28S rRNA cleavage. MHV-induced 28S rRNA cleavage was independent of virus-induced CPE. and it differed from apoptosis-related rRNA degradation, in that blocking apoptosis had no effect on the cleavage of 28S rRNA.

MHV-induced 28S rRNA cleavage could occur via the activation of a cellular or viral RNase. That same RNase may degrade host mRNAs, which maybe partly responsible for the host protein shut-off in MHV-infected cells (Hilton et al 1986). Alternatively, binding of MHV factors or host factors may alter the structure of $60 \mathrm{~S}$ ribosome, allowing a cellular or viral RNase to access specific regions of $28 \mathrm{~S}$ rRNA and cleave it.

$28 \mathrm{~S}$ rRNA being an integral component of the $60 \mathrm{~S}$ ribosomal subunit, MHV-induced 28S rRNA cleavage may affect protein synthesis by rendering polysomes, containing cleaved $28 \mathrm{~S}$ rRNA, translationally inactive. Polysomes with intact $28 \mathrm{~S}$ rRNA maybe used for MHV-specific protein synthesis, resulting in host mRNA translation inhibition. Alternatively, ribosomes, containing $28 \mathrm{~S}$ cleavage products, maybe structurally altered to better translate increasing amounts of MHV-specific mRNAs. Thus, MHVinduced 28S rRNA cleavage may alter the cellular environment and 
translation machinery to slow down host protein synthesis and allow efficient expression of viral proteins.

\section{ACKNOWLEDGMENTS}

This work was supported by Public Health Service grant AI29984 from the National Institutes for Health.

\section{REFERENCE}

An, S., Chen, C.-J., Yu, X., Leibowitz, J. L., and Makino, S., 1999, Induction of apoptosis in murine coronavirus-infected cultured cells and demonstration of $E$ protein as an apoptosis inducer. J. Virol. 73:7853-7859.

Dveksler, G. S., Pensiero, M. N., Cardellichio, C. B., Williams, R. K., Jiang, G.-S., Holmes, K. V., and Dieffenbach, C. W., 1991, Cloning of the mouse hepatitis virus (MHV) receptor: expression in human and hamster cell lines confers susceptibility to MHV. J. Virol. 65:6881-6891.

Hilton, A., Mizzen, L., Macintyre, G., Cheley, S., and Anderson, R., 1986, Translational control in murine hepatitis virus infection. J. Gen. Virol. 67:923-932.

Kim, K.-H., Narayanan, K., and Makino, S., 1997, Assembled coronavirus from complementation of two defective interfering RNAs. J. Virol. 71:3922-3931.

Kyuwa, S., Cohen, M., Nelson, G.W., Tahara, S. M., and Stohlman, S. A., 1994, Modulation of cellular macromolecular synthesis by coronavirus:Implications for pathogenesis. $J$. Virol. 68:6815-6819.

Lai, M. M. C., Brayton, P. R., Armen, R. C., Patton, C. D., Pugh, C., and Stohlman, S. A., 1981, Mouse hepatitis virus A59: mRNA structure and genetic localisation of the sequence divergence from hepatotropic strain MHV-3. J. Virol. 39:823-834.

Lee, H.-J., Shieh, C.-K., Gorbalenya, A. E., Koonin, E. V., La Monica, N., Tuler, J., Bagdzhadzhyan, A., and Lai, M. M. C., 1991, The complete sequence (22 kilobases) of murine coronavirus gene 1 encoding the putative proteases and RNA polymerase. Virology 180:567-582.

Makino, S., Joo, M., and Makino, J. K., 1991, A system for study of coronavirus mRNA synthesis: a regulated, expressed subgenomic defective interfering RNA results from intergenic site insertion. J. Virol. 65:6031-6041.

Sawicki, S. G., and Sawicki, D. L., 1986, Coronavirus minus-strand RNA synthesis and effect of cyclohexamide on coronavirus RNA synthesis. J.Virol. 57:328-334.

Siddell, S., Wege, H., Barthel, A., and ter Meulen, V., 1981, Coronavirus JHM: Intracellular protein synthesis. J. Gen. Virol. 53:145-155.

Tahara, S. M., Dietlin, T. A., Bergmann, C. C., Nelson, G. W., Kyuwa, S., Anthony, R. P., and Stohlman, S. A., 1994, Coronavirus translation regulation: leader affects mRNA efficiency. Virology 202:621-630.

Tahara, S. M., Dietlin, T. A., Nelson, G. W., Stohlman, S. A., and Manno, D. J., 1998, Mouse hepatitis virus nucleocapsid protein as a translational effector of viral mRNAs. $A d v$. Exp. Med. Biol. 440:313-318. 